\title{
Editorial: Deep Learning for Big Data Analytics
}

\author{
Yulei $\mathrm{Wu}^{1} \cdot \mathrm{Fei} \mathrm{Hao}^{2} \cdot$ Sambit Bakshi ${ }^{3} \cdot$ Haojun Huang ${ }^{4}$
}

Accepted: 29 September 2021 / Published online: 10 November 2021

(c) The Author(s), under exclusive licence to Springer Science+Business Media, LLC, part of Springer Nature 2021

\section{Contents}

Noushin Jafarpisheh, Effat J. Zaferani, Mohammad Teshnehlab, Hadis Karimipour, Reza M. Parizi, and Gautam Srivastava, "A Deep Neural Network Combined with Radial Basis Function for Abnormality Classification", Mobile Networks and Applications, this issue.

Qindong Sun, Xingyu Feng, Shanshan Zhao, Han Cao, Shancang Li, Yufeng Yao. Deep learning-based Customer Preferences Analysis in Industry 4.0 Environment. Mobile Networks and Applications, this issue.

Zhen Pang, Xiang Wang, Xulong Wang, Jun Qi, Zhong Zhao, Yuan Gao, Yun Yang, Po Yang, A Multi-modal Data Platform for Diagnosis and Prediction of Alzheimer's Disease Using Machine Learning Methods, Mobile Networks and Applications, this issue.

Eric Ke Wang, Zhiyuan Tan, Kuo-Hui Yeh, Xunguang Wang, Peng Xu, Chien-Ming Chen, GAN based Text Data Augmentation for IoT domain Dialogue System, Mobile Networks and Applications, this issue.

Zhaoxue Jiang et al. Survey on Log Research of AIOps: Methods and Trends, Mobile Networks and Applications, this issue.

Roopali Dogra, Shalli Rani, Sandeep Verma, Sahil Garg, Mohammad Mehedi Hassan, TORM: Tunicate Swarm Algorithm-based Optimized Routing Mechanism in IoT-based framework, Mobile Networks and Applications, this issue.

Yulei Wu

y.l.wu@exeter.ac.uk

1 College of Engineering, Mathematics and Physical Sciences, University of Exeter, Exeter EX4 4QF, UK

2 School of Computer Science, Shaanxi Normal University, Xi'an 710119, China

3 Department of Computer Science \& Engineering, National Institute of Technology Rourkela, Odisha 769008, India

4 School of Electronic Information and Communications, Huazhong University of Science and Technology, Wuhan 430074, China
Carlos Luis González-Valiente, Magda León Santos, Ricardo Arencibia-Jorge, Ed Noyons, Rodrigo Costas, Mapping the evolution of intellectual structure in information management using author co-citation analysis, Mobile Networks and Applications, this issue.

\section{Editorial}

With the fast-growing data deluge across all complex realworld applications such as social networks, biological networks, Internet of Things (IoT), and so on, the current big data has exhibited unstructured, heterogeneous, multisource, sheer volume and complex natures. These emerging characteristics pose great challenges on the existing data processing techniques. Deep learning is currently a promising technology on data analysis in a number of key application areas, such as speech recognition, computer vision, and natural language processing. It is coming to play an important role in coping with the challenges of big data and providing effective big data analytics solutions [1-5]. This special issue is devoted to the most recent developments and research outcomes addressing the related theoretical and practical aspects on deep learning for big data analytics, and it also aims to provide worldwide researchers and practitioners an ideal platform to innovate new solutions targeting at the corresponding key challenges.

Working on cancer datasets encounters researchers to two problems. The first is the large dimension of features and low numbers of samples, and the second is the existing uncertainties, noise, and the requirement for overcoming them. In the context of big data, deep learning has important applications in a wide range of fields, especially, in bioinformatics and cancer classification to address the above-mentioned issues. Accordingly, the authors in the paper entitled "A Deep Neural Network Combined with Radial Basis Function for Abnormality Classification" [6], proposed the use of Radial Basis Function (RBF) as the nonlinear function of Deep Neural Network (DNN). In spite of the traditional structure of DNN, the proposed approach has only one 
weight matrix to be trained. Furthermore, applying the Gaussian activation function prevents potential problems, such as untrained matrix weights which result in stopping the training phase.

Deep learning techniques have achieved excellent performance in data analysis applications. In view of this, the authors in the paper entitled "Deep learning-based Customer Preferences Analysis in Industry 4.0 Environment" [7], used deep learning to analyze the real sales data of the catering industry to clarify customer preferences and potential characteristics. The authors split the original time series into normal working day data and holiday data, and then establish a dishes sales forecasting model using deep learning. The results show that the proposed forecasting model has better predictive performance and strong robustness. It is a successful application of deep learning for big data analysis of catering industry and has great development potential.

The paper entitled "A Multi-modal Data Platform for Diagnosis and Prediction of Alzheimer's Disease Using Machine Learning Methods" [8], used a multi-task learning algorithm based on the ADNI dataset to predict the cognitive scores of subjects in the next three years. This method effectively assesses the cognitive trends of patients and predicts the progression of the disease. Also, the authors use four different machine learning classification algorithms to conduct fusion research on AD multi-modal data, and thus to achieve the effect of assisting doctors in diagnosis. Finally, the authors design a multi-modal data platform technical architecture to standardize management and sharing of ADNI data and the data obtained by offline medical institutions to improve the utilization and value of data.

Data augmentation is an efficient way to overcome the deficiency problem of labeled data sets for training. The authors in the paper entitled "GAN based Text Data Augmentation for IoT domain Dialogue System" [9], proposed a GAN-based text augmentation model to augment data for realizing a dialogue system in an IoT system. The text augmentation model is compared with the basic text generation model, the Recurrent Neural Network-based text generation model, and the RankGAN text generation model on the data sets of the ultrasonic inspection reports and the novels. On the two data sets, the proposed model has achieved better results.

Log with the characteristics of large-scale, rich types, complex structure, and uneven qualities, is one of the most useful data resources in IT system, and plays an important role in Artificial Intelligence for IT Operations (AIOps). The paper entitled "Survey on Log Research of AIOps: Methods and Trends" [10], reviewed and categorized the log research on enhancing log quality, analyzing log structure, understanding system behavior, and helping users mine the effective information by machine learning and deep learning. This paper also establishes evaluation indicators for comparison and summary, and discusses the potential directions and future development trends in log research.

The paper entitled "TORM: Tunicate Swarm Algorithm-based Optimized Routing Mechanism in IoT-based framework" [11], presented Tunicate Swarm Algorithm (TSA)-based Optimized Routing Mechanism (TORM) that addresses the problem of energy-efficiency of sensor nodes for IoT for longer sustainability. It is revealed through the simulation analysis that TORM outperforms various stateof-the-art algorithms used for optimized selection of cluster head.

The current big data era has placed new challenges for the study and application of information management (IM). In this paper entitled "Mapping the evolution of intellectual structure in information management using author co-citation analysis" [12], the authors visualized how IM evolved over thirty-six years (1980-2015), and provided comprehensive bibliometric research oriented to visualize the overall evolution IM. Several important findings are observed and analyzed in the paper.

Acknowledgements We would like to express our deep thanks to the Editor-in-Chief, Professor Imrich Chlamtac, for providing us with the opportunity to host this special issue in ACM/Springer Mobile Networks \& Applications (MONET). We also thank all the authors who submitted their papers. Last but not least, we thank the thoughtful work of the many reviewers who have provided invaluable evaluations and recommendations.

\section{References}

1. Yan Z, Ge J, Wu Y, Li L, Li T (2020) Automatic virtual network embedding: A deep reinforcement learning approach with graph convolutional networks. IEEE J Sel Areas Commun 38(6):1040 1057. https://doi.org/10.1109/JSAC.2020.2986662

2. Wang H, Wu Y, Min G, Miao W. A Graph Neural Network-based Digital Twin for Network Slicing Management, IEEE Transactions on Industrial Informatics. https://doi.org/10.1109/TII.2020. 3047843

3. Wu Y, Dai H-N, Tang H. Graph neural networks for anomaly detection in industrial internet of things. IEEE Internet Things J. https://doi.org/10.1109/JIOT.2021.3094295

4. Wu Y, Wang Z, Ma Y, Leung VCM (2021) Deep reinforcement learning for blockchain in industrial IoT: A survey. Comput Netw 191:108004. https://doi.org/10.1016/j.comnet.2021.108004

5. Wu Y (2021) Cloud-edge orchestration for the internet of things: architecture and aI-powered data processing. IEEE Internet Things J 8(16):12792-12805. https://doi.org/10.1109/JIOT.2020.3014845

6. Jafarpisheh N, Zaferani EJ, Teshnehlab M, Karimipour H, Parizi RM, Srivastava G. "A Deep Neural Network Combined with Radial Basis Function for Abnormality Classification”, Mobile Networks and Applications, this issue

7. Sun Q, Feng X, Zhao S, Cao H, Li S, Yao Y. Deep learning-based Customer Preferences Analysis in Industry 4.0 Environment. Mobile Networks and Applications, this issue

8. Pang Z, Wang X, Wang X, Qi J, Zhao Z, Gao Y, Yang Y, Yang P. A Multi-modal Data Platform for Diagnosis and Prediction of Alzheimer's Disease Using Machine Learning Methods, Mobile Networks and Applications, this issue 
9. Wang EK, Tan Z, Yeh K-H, Wang X, Xu P, Chen C-M. GAN based Text Data Augmentation for IoT domain Dialogue System, Mobile Networks and Applications, this issue

10. Jiang X et al. Survey on Log Research of AIOps: Methods and Trends, Mobile Networks and Applications, this issue

11. Dogra R, Rani S, Verma S, Garg S, Hassan MM. TORM: Tunicate Swarm Algorithm-based Optimized Routing Mechanism in IoTbased framework, Mobile Networks and Applications, this issue

12. González-Valiente CL, Santos ML, Arencibia-Jorge R, Noyons $\mathrm{E}$, Costas R. Mapping the evolution of intellectual structure in information management using author co-citation analysis, Mobile Networks and Applications, this issue

Publisher's Note Springer Nature remains neutral with regard to jurisdictional claims in published maps and institutional affiliations. 